\title{
PROPOSTA DE METODOLOGIA HÍBRIDA PARA DETERMINAÇÃO DE CAPACIDADE DE HOSPEDAGEM DE FONTES DE GERAÇÃO FOTOVOLTAICA EM REDES DE DISTRIBUIÇÃO
}

\author{
Ezequiel Junio de Lima ${ }^{1}$, Luiz Carlos Gomes de Freitas ${ }^{2}$ \\ ${ }^{1}$ Instituto Federal do Sul de Minas (IFSULDEMINAS) - Campus Poços de Caldas, Poços de Caldas - MG, Brasil \\ ${ }^{2}$ Universidade Federal de Uberlândia (UFU), Uberlândia - MG, Brasil \\ e-mail: ezequiel.lima@ifsuldeminas.edu.br, lcgfreitas@ufu.br
}

\begin{abstract}
Resumo - O conceito de capacidade de hospedagem (CH) está se tornando mais difundido, já que é uma abordagem abrangente que permite entender os impactos da geração distribuída nas redes. Vários métodos de cálculo da $\mathrm{CH}$ foram desenvolvidos nos últimos anos e são classificados em determinísticos, estocásticos e séries temporais, cada qual com os seus respectivos prós e contras. Dentro desse contexto, neste artigo é proposta uma metodologia híbrida que combina os métodos determinísticos e de séries temporais, no cálculo da $\mathbf{C H}$ de um alimentador real, situado em Poços de Caldas, MG, da concessionária DMED. O referido alimentador foi modelado e analisado levando-se em consideração variações de cargas e de potência injetada por fontes GD nas diferentes barras que compõem o sistema. Por fim, os resultados alcançados demonstram a eficácia da metodologia proposta.
\end{abstract}

Palavras-chave - Capacidade de Hospedagem, Energia Fotovoltaica, Geração Distribuída, Redes de Distribuição.

\section{PROPOSAL OF A HYBRID METHODOLOGY FOR HOSTING CAPACITY ANALYSIS}

\begin{abstract}
The concept of hosting capacity (HC) is becoming more widespread, as it is a comprehensive approach that allows understanding the impacts of distributed energy resources into the distribution system. Several methods for calculating $\mathrm{HC}$ have been developed in recent years and are classified into deterministic, stochastic and time series, each with its respective pros and cons. Within this context, in this paper the authors present a hybrid methodology that combines deterministic and time series methods to find a real feeder hosting capacity, located in "Poços de Caldas" city, Brazil, from the utility company "DMED". This feeder was modeled and analyzed taking into account variations in loads and power injection by DER's on the system's buses. Finally, the results achieved demonstrate the effectiveness of the proposed methodology.
\end{abstract}

Keywords - Distributed Energy Resources, Distribution Grids, Hosting Capacity, Photovoltaic Energy.

Manuscript received 09/09/2020; first revision 12/02/2020; accepted for publication 01/15/2021, by recommendation of Editor Demercil de Souza Oliveira Jr. http://dx.doi.org/10.18618/REP.2021.1.0057

\section{NOMENCLATURA}

$\mathrm{CH} \quad$ Capacidade de Hospedagem.

$G D$ Geração Distribuída.

$U F V \quad$ Usina Fotovoltaica.

\section{INTRODUÇÃO}

Nos últimos 10 anos, a energia solar fotovoltaica tem mostrado potencial para tornar-se uma das fontes de eletricidade predominantes no mundo, com um crescimento robusto e contínuo mesmo em tempos de crise financeira e econômica. A Agência Internacional de Energia (IEA) estima que a energia solar se tornará uma das principais fontes de energia até 2050 e contribuirá com cerca de $11 \%$ da geração de energia mundial [1].

O Brasil está seguindo a tendência mundial e apresentase como mercado promissor. Segundo o balanço energético nacional de 2019, houve um aumento da geração elétrica solar fotovoltaica de $316,1 \%$ do ano de 2017 para 2018, representando um salto de $832 \mathrm{GWh}$ para $3461 \mathrm{GWh}$ [2]. Com isso, a participação do modal solar na matriz elétrica brasileira, em 2019, chegou a $0,5 \%$ do total [2] e, em agosto de 2020, a 1,6\% [3]. Se consideradas a geração distribuída (GD) e a geração centralizada (GC), até novembro de 2020, o Brasil registrou a potência instalada operacional de 7,00 GW [3].

Alinhada com este panorama e atenta ao mercado, a Agência Nacional de Energia Elétrica (ANEEL) lança, em 2016, a Chamada $N^{\circ} .001 / 2016$ Projeto Prioritário de Eficiência Energética e Estratégico de P\&D: "Eficiência Energética e Minigeração em Instituições Públicas de Educação Superior" [4], a fim de fomentar parcerias entre Concessionárias e Universidades Públicas. O objetivo do edital foi facilitar a inserção da geração fotovoltaica na matriz energética brasileira e no setor público, buscando avaliar a viabilidade econômica da geração própria de energia e seus impactos nos sistemas de distribuição [4].

Logo, tem-se, por um lado, o crescimento da fonte renovável fotovoltaica que traz benefícios ao consumidor e contribui para: segurança do fornecimento de energia elétrica; modicidade tarifária; diminuição do impacto ambiental do setor elétrico e redução da dependência da hidrogeração. Por outro, leva novas preocupações às concessionárias de energia quanto a disseminação de GD na sua rede e os impactos que o aumento da geração fotovoltaica pode trazer.

Em seu trabalho, Karimi et al. [5] aponta que devido à popularização de usinas fotovoltaicas (UFVs), se faz necessário realizar estudos para investigar possíveis impactos 
na qualidade do "produto energia elétrica", coordenação e operação da proteção das redes de distribuição. Um exemplo recente, pode ser encontrado no trabalho de Reinaldo, Dupczak e Aranha Neto [6] para a rede de baixa tensão da concessionária CELESC.

Desta forma, os estudos nessa área de avaliação de problemas oriundos da conexão de geração distribuída na rede de distribuição utilizam a $\mathrm{CH}$ e/ou limite de sustentabilidade para definir a capacidade máxima da fonte de energia solar fotovoltaica que pode ser conectada a uma determinada rede, ou a um determinado alimentador, sem transgredir um parâmetro regulatório, isto é, sem resultar em parâmetros inaceitáveis de confiabilidade, de qualidade de energia e/ou de segurança [7], [8].

Segundo a EPRI [9], o conceito de $\mathrm{CH}$ não é novo, mas sua aplicação está se tornando mais difundida, pois o mercado precisa de uma abordagem abrangente para entender os impactos da GD. Assim, vários métodos de determinação da $\mathrm{CH}$ foram desenvolvidos nos últimos anos [10]-[12], resultando em um debate considerável sobre "qual", "como" e "quando" tais métodos devem ser usados.

Neste aspecto, os estudos reportados em [9], [11], [12] apresentam os principais métodos empregados para o cálculo da $\mathrm{CH}$ e os classificam em determinísticos, estocásticos e séries temporais.

Diante deste cenário, os autores propõem uma metodologia híbrida que se mostra eficaz e de fácil implementação. Para validação do método proposto, são apresentados os resultados do estudo que teve como objetivo determinar a $\mathrm{CH}$ de um alimentador real situado no município de Poços de Caldas, MG, da concessionária DME Distribuição (DMED). $\mathrm{O}$ referido alimentador foi modelado e analisado levando-se em consideração variações de cargas e de potência injetada por fontes GD nas diferentes barras que compõem o sistema.

\section{MÉTODOS DE DETERMINAÇÃO DA CH: ESTADO DA ARTE}

\section{A. Método Determinístico}

Os métodos determinísticos aplicam dados de entrada conhecidos e fixos a um modelo da rede de distribuição para analisar os impactos da inserção de energia solar fotovoltaica. Assim, sua implementação é simples e a rede de distribuição é modelada usando potência ativa $(\mathrm{P})$, potência reativa $(\mathrm{Q})$, impedâncias série de linha $(\mathrm{Z})$ e modelos de carga com impedância, corrente ou potência constante. Avaliam o sistema com base em cenários e normalmente consideram o pior caso possível para avaliar o impacto extremo de parâmetros predefinidos, tal como a entrada de uma usina fotovoltaica [9], [12], [13].

O método emprega a análise tradicional de fluxo de potência como ferramenta para os cálculos. Sua implementação é considerada simples por ser uma ferramenta corriqueira nas análises empregadas pela maioria das concessionárias [12]. Nos resultados, apresenta uma visão geral e rápida do desempenho da rede com a conexão de geração distribuída (GD). Como são assumidos valores fixos, não considera a natureza intermitente da GD fotovoltaica e, portanto, a $\mathrm{CH}$ obtida é uma estimativa do pior cenário. Consequentemente, o impacto tende a ser superestimado e a
CH subestimada [11].

\section{B. Métodos Estocásticos}

Ao se modelar uma rede de distribuição com GD, é comum deparar-se com parâmetros incertos. Por exemplo, a produção de energia fotovoltaica de uma determinada UFV é incerta, afinal depende da irradiação solar que, por sua vez, é influenciada pelas mudanças nas condições climáticas. Além dessas incertezas, a demanda dos consumidores, o tamanho das UFVs que serão adicionadas à rede, suas respectivas localizações geográficas e demais características técnicas e construtivas são muitas vezes desconhecidas [12],[13]. Assim, os métodos estocásticos consideram a chance de ocorrência e as variações presentes nas redes de distribuição em meio a essas incertezas e culminam em um fluxo de potência probabilístico.

Assim, para a entrada de dados desse método de cálculo, faz-se necessário definir quais incertezas serão consideradas e quais funções estatísticas de densidade de probabilidade melhor as representa, sendo comum o emprego da distribuição uniforme, de Poisson, de Gauss, Binomial dentre outras. Consequentemente, as variáveis de saída também serão dadas por uma função de densidade de probabilidade. A avaliação e interpretação destes resultados não é trivial e, portanto, a determinação da CH torna-se uma tarefa difícil [12], [14], [15].

Para Kharrazi, Sreeram e Mishra [13], é a técnica que melhor modela as incertezas e aleatoriedades da rede. Entretanto, requer um grande tempo e esforço computacional à medida que o modelo da rede, ou o número de incertezas consideradas, cresce.

Mulenga, Bollen e Etherden [12] afirmam que se o objetivo do estudo é o dimensionamento da rede, haverá pouco valor agregado em considerar uma grande quantidade de cenários com condições moderadas, isto é, bastaria a análise do pior caso. Os métodos estocásticos, portanto, nem sempre são necessários.

\section{Método de Séries Temporais}

Os métodos de séries temporais utilizam medições reais do sistema como entrada para os cálculos de $\mathrm{CH}$. Desta maneira são empregadas "curvas de cargas", "curvas de produção de energia fotovoltaica", "perfil de tensão do alimentador", dentre outras, para a simulação da rede em cada momento desejado.

Por usar dados medidos em relação ao tempo, o método permite fazer correlações entre as variáveis, como por exemplo, o aumento de temperatura com o aumento do consumo de energia e aumento e/ou redução da produção de energia fotovoltaica. Permite também uma visão realista do comportamento da rede com GD, uma vez que produção de energia fotovoltaica e o consumo de energia por parte dos usuários são características naturalmente variáveis no tempo.

Sua implementação é complexa pois requer muitos dados de medições para a construção das séries temporais. A coleta deste dados não é algo trivial e requer que a concessionária tenha em sua estrutura, ou uma equipe, ou instrumentos automatizados, para coleta e análise destas informações. Via de regra, as concessionárias de energia fazem "Campanhas de Medição", onde equipes vão à campo para instalação e coleta de informações, ou ainda, através de parcerias com a Academia via projetos de $\mathrm{P} \& \mathrm{D}$, por exemplo. De forma 
alternativa, caso não se tenha uma série histórica dos dados, ou a disponibilidade de equipes, pode-se utilizar de processos estocásticos para fabricar os dados de séries temporais para um período de tempo mais longo, tais como os métodos Autorregressivos (AR - autoregressive), Autorregressivos de Médias Móveis (ARMA - autoregressive moving average) e Autorregressivos Integrados de Médias Móveis (ARIMA autoregressive integrated moving average) [12].

Deve-se atentar ainda para qual parâmetro se deseja fazer os estudos, pois isso influenciará diretamente na resolução temporal da coleta de dados. Por exemplo, para variações de tensão de longa duração, uma resolução de 15 minutos podem ser suficientes, no entanto, variações de tensão de curta duração podem requerer uma resolução temporal de 1 minuto (ou menos). Dificultando, portanto, o armazenamento e processamento desses dados a medida que o tempo de coleta aumenta.

De forma sucinta, a Tabela I traz os principais indicadores que podem determinar a escolha e aplicação de cada metodologia apresentada.

\section{METODOLOGIA HÍBRIDA PROPOSTA}

Ao analisar cada método apresentado na seção anterior, depreende-se que o método determinístico para o cálculo de $\mathrm{CH}$, embora rápido e de fácil emprego, não leva em consideração incertezas tais como produção de energia fotovoltaica, a demanda dos consumidores, o tamanho da UFV etc. Por sua vez, o método estocástico trata as incertezas mas não considera as relações temporais das grandezas estudadas impedindo estudos de coordenações de proteção ou a correlação entre produção e demanda de energia.

Por fim, o método que emprega séries temporais, lida com as variações de produção de energia fotovoltaica e com as variações de demanda dos consumidores, embora requeira dados históricos de medições para construir as séries de tempo de forma que permita a análise de comportamento das cargas. Isso implica deslocamento de equipes para instalação de medidores na rede e, consequentemente, em custos.

Para obter um método híbrido, optou-se por combinar os métodos determinísticos e de séries temporais, a fim de tirar o melhor proveito de ambos. Para tal, na modelagem do alimentador são utilizadas séries temporais de demanda e tensão de forma a levar em consideração as variações temporais das cargas e da injeção de energia da UFV, tal qual seria feito no método de série temporal típico. A coleta destas curvas que pode ser feita diretamente na subestação de origem do alimentador, haja visto que é comum as concessionárias instalarem nos alimentadores de média tensão medidores/registradores de potência, tensão e corrente etc. A forma de coleta de dados, por si só, já representa uma vantagem que permite o uso do método de série temporal devido a facilidade de obtenção.

As cargas de baixa tensão são modeladas pela razão entre suas demandas máximas e a demanda máxima do alimentador. Isto é, as cargas são definidas de forma determinística e cada carga contribui proporcionalmente com a demanda no alimentador, conforme mostram as equações (1) e (2)

$$
\begin{gathered}
\alpha_{1} \cdot D_{A L M}(t)+\alpha_{2} \cdot D_{A L M}(t)+\cdots+ \\
+\alpha_{n} \cdot D_{A L M}(t)=D_{A L M}(t) \\
\alpha_{i}=\left.\frac{\max \left(D_{\text {carga }}\right)}{\max \left(D_{A L M}\right)}\right|_{i=1,2, \cdots, n} .
\end{gathered}
$$

Onde:

$\begin{array}{ll}D_{A L M} & \text { Demanda no alimentador; } \\ \alpha_{i} & \text { Coeficiente de contribuição da carga } i ; \\ n & \text { Número de cargas. }\end{array}$

Dessa forma o sistema pode ser simulado de acordo com o comportamento típico da rede, formando cenários mais realistas. A velocidade de processamento e tempo computacional será um valor intermediário entre ambos. Sua implementação é mais simples por executar apenas os casos de interesse para o estudo em questão.

Em suma, pode-se elencar as seguintes vantagens proporcionadas pelo método híbrido proposto neste trabalho:

* Obtenção dos parâmetros de entrada facilitada;

* Agilidade na implementação do estudo;

* Maior velocidade no processamento;

* Resultados coerentes com o método temporal, conforme mostrado na seção VI.

Portanto, dentro desse contexto, este artigo busca contribuir

TABELA I

Comparação entre Metodologias de Cálculo de Hospedagem

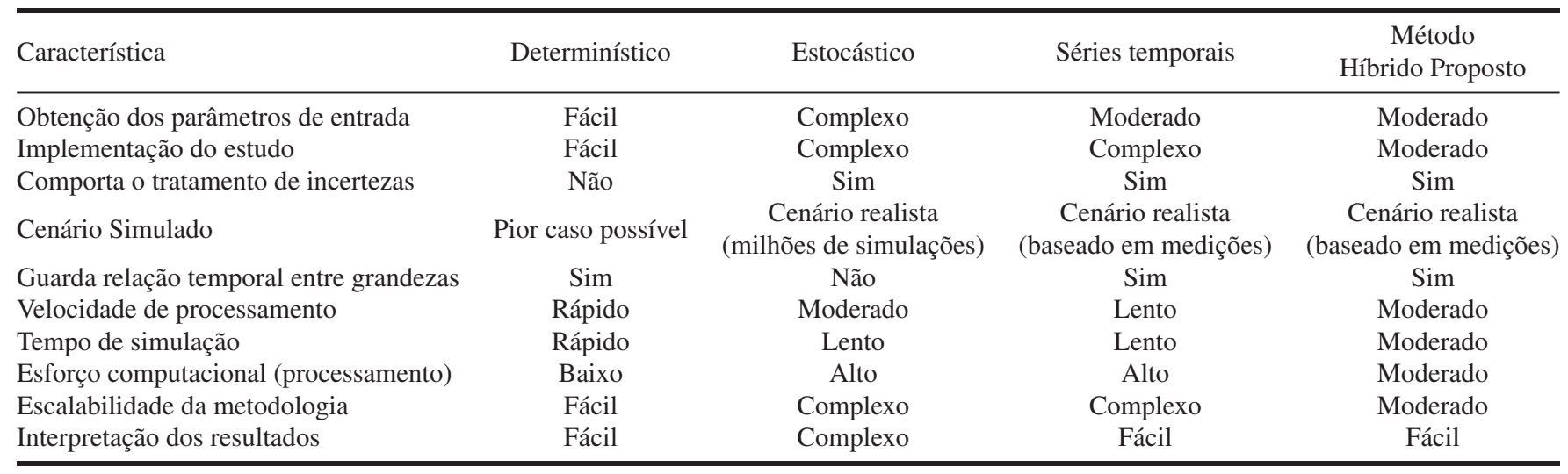


ao tema, aplicando a metodologia híbrida proposta no cálculo da $\mathrm{CH}$ de um alimentador real situado no município de Poços de Caldas, MG, da concessionária DME Distribuição (DMED).

\section{MODELO DO SISTEMA EM ESTUDO}

O sistema em estudo é composto por um alimentador radial de média tensão, uma miniusina fotovoltaica (miniUFV) e diversas cargas que podem ser observados no diagrama unifilar apresentado na Figura 1. O circuito alimentador 19 supre a Zona Sul do município de Poços de Caldas, MG, se estende por cerca de $22 \mathrm{~km}$ atendendo 7084 instalações consumidoras (até a presente data). Ademais, opera com uma tensão nominal de $13,8 \mathrm{kV}$ e parte de um trafo de 20 MVA da subestação Saturnino de Brito $\left(S E_{P C 3}\right)$. A construção do modelo das cargas e da UFV é explicitada nas subseções seguintes e as informações dos demais componentes (cabos, capacitores etc.) foram cedidas pela DME Distribuição.

A modelagem na metodologia híbrida foi realizada através do software comercial SSEP ${ }^{\circledR}$ versão 4.0.0.25. O programa usa técnicas do domínio da frequência para a solução de fluxos de carga convencional e harmônico dos sistemas elétricos de potência [16].

A título de comparação e validação, para o cálculo usando séries temporais, o sistema também foi modelado empregando o software gratuito OpenDSS ${ }^{\mathrm{TM}}$ [17].

\section{A. Cargas Lineares}

Segundo Kagan, Oliveira e Robba [18], dentre todos os componentes do sistema elétrico, talvez os que apresentem um maior desafio para modelagem sejam as cargas. Para sua representação, é comum a utilização de cargas concentradas em determinados pontos da rede, cargas uniformemente distribuídas, cargas representadas pela demanda máxima e cargas representadas através de curvas típicas.

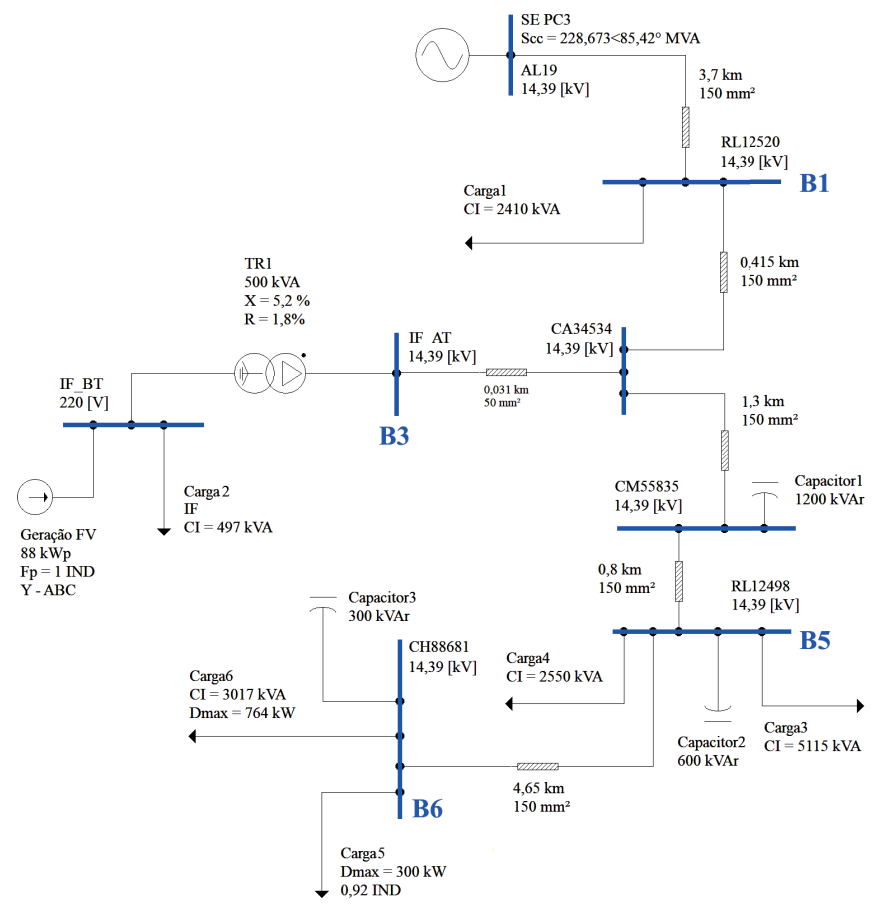

Fig. 1. Diagrama unifilar do alimentador 19.
Adotou-se então duas formas distintas para a representação das cargas. No modelo onde será empregado a técnica de séries temporais, as cargas de baixa tensão serão representadas por curvas típicas. No modelo empregado para a técnica híbrida, as cargas de baixa tensão serão representadas pela demanda máxima, conforme (1). A Tabela II apresenta os valores dos coeficientes de contribuição de cada carga, calculados por (2). Em ambos modelos, as cargas foram agrupadas de acordo com os bairros atendidos conforme podese ver indicado na Figura 1.

TABELA II

Coeficientes de Contribuição das Cargas do Método Híbrido Proposto

\begin{tabular}{ccccccc}
\hline & Carga $_{1}$ & Carga $_{2}$ & Carga $_{3}$ & Carga $_{4}$ & Carga $_{5}$ & Carga $_{6}$ \\
\hline$\alpha_{i}$ & $10,3 \%$ & $1,1 \%$ & $33,7 \%$ & $26,9 \%$ & $10,1 \%$ & $17,9 \%$ \\
\hline
\end{tabular}

O comportamento da rede de média tensão foi modelada através da curva de carga com a coleta de dados sendo realizada por um registrador de demanda e energia, instalado na subestação $\left(S E_{P C 3}\right)$, guardando valores de tensões, correntes, energia e demanda durante o período de janeiro a julho do ano de 2019, com um intervalo de integração de 5 minutos.

Os resultados compilados para a demanda são apresentados na Figura 2. Pode-se observar que seu comportamento é típico de consumidores de caráter predominantemente residencial conforme indicam os estudos [19]-[21].

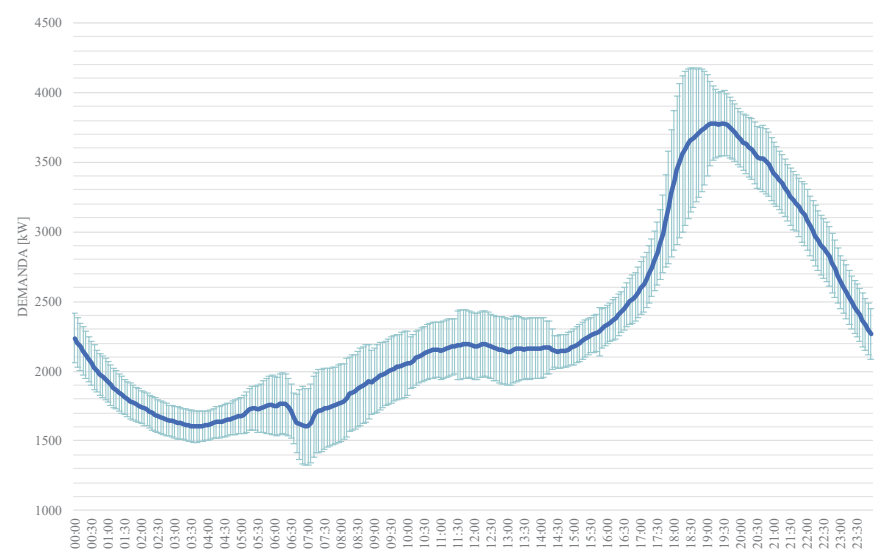

Fig. 2. Média das demandas diárias entre janeiro e julho de 2019 e desvio padrão associado do Alimentador 19.

\section{B. Usina Fotovoltaica}

A inclusão de GD no alimentador 19 tomará como base a usina fotovoltaica (UFV) já existente no alimentador, situada no campus Poços de Caldas do IFSULDEMINAS, instalada desde janeiro de 2017, com $88 \mathrm{kWp}$ de potência nominal.

Segundo Sauthier et al. [22], um problema recorrente para modelar aplicações fotovoltaicas é a previsão do comportamento elétrico de um gerador fotovoltaico, dado serem necessárias as informações construtivas dos módulos fotovoltaicos, a localização geográfica e as características do clima local que influenciam diretamente a produção de energia. Para contornar esta dificuldade, o gerador fotovoltaico será modelado como uma fonte de corrente e o 


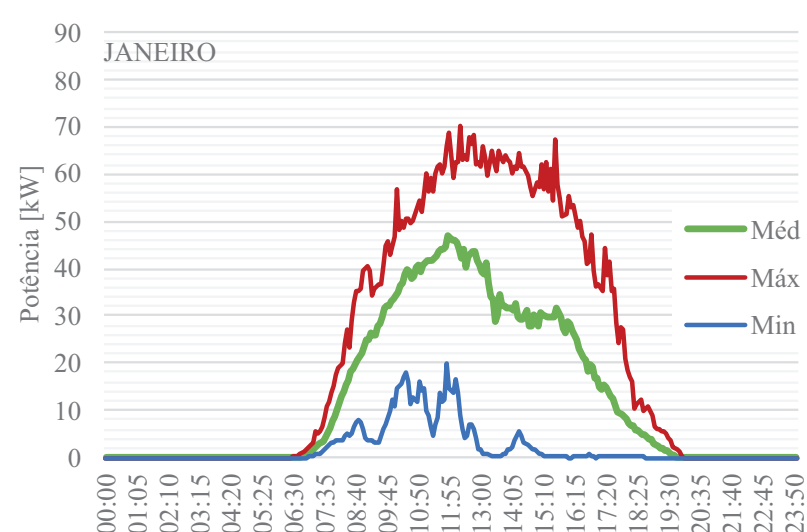

ठ்
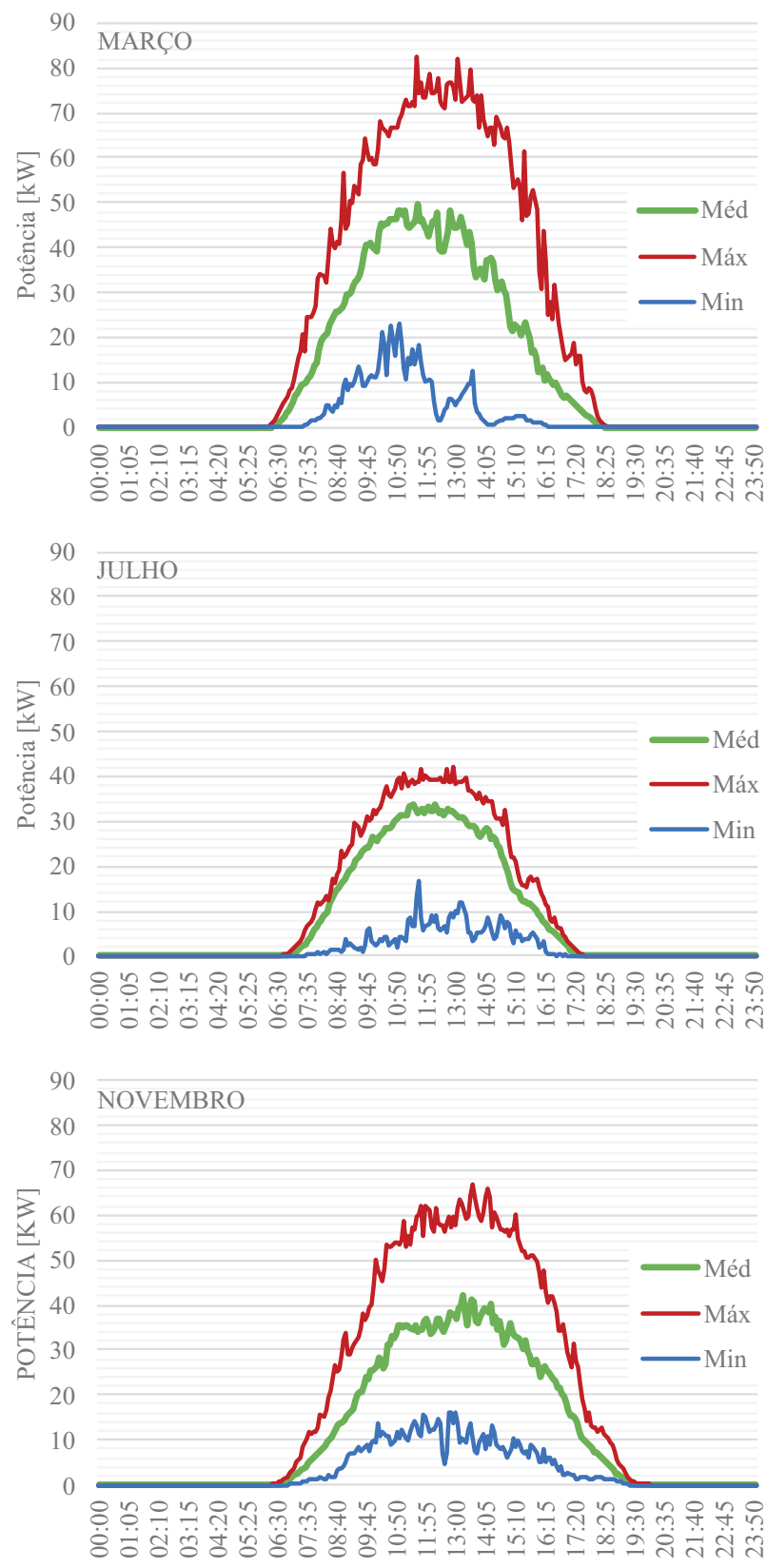

Fig. 3. Produção média horária de janeiro, março, julho e novembro de 2019 da UFV do IFSULDEMINAS Campus Poços de Caldas. Pode-se ver a média horária mensal e os valores máximos e mínimos registrados no período. valor de injeção de corrente variará conforme o caso a ser simulado, seguindo as curvas de produção mensal média da UFV. A Figura 3 mostra alguns exemplos dessas curvas de produção de energia para os meses de janeiro, março, julho e novembro de 2019.

\section{Ajuste e Calibração do Modelo}

Para avaliar os modelos, optou-se por realizar o confrontamento das simulações com as medições realizadas em campo. Assim, foram registrados de forma simultânea demandas, geração de energia solar fotovoltaica e os níveis de tensões tanto na saída da subestação Saturnino $\left(S E_{P C 3}\right)$ quanto na entrada da subestação do IFSULDEMINAS ( $\left.S E_{I F}\right)$. Escolheu-se então uma quarta-feira (16.01.2019), um sábado (26.01.2019) e o dia 17.07.2019 (uma quarta-feira com o maior registro de demanda no período avaliado).

A Tabela III apresenta os dados obtidos com as medições e simulações, nos dias 16 e 26 de janeiro, apenas para o modelo do método híbrido. Destaca-se que nos casos 01 e 02, o modelo da rede foi configurado para o pico de geração fotovoltaica e, nos demais, para o pico de demanda do alimentador. Ainda na Tabela III, $P_{F V}$ indica a potência instantânea da usina fotovoltaica; $D_{I F}$ e $D_{A L 19}$ são, respectivamente, as demandas do IFSULDEMINAS e do alimentador 19; $V_{S E_{P C 3}}$ e $V_{S E_{I F}}$ são as tensões nos barramentos das subestações Saturnino $\left(S E_{P C 3}\right)$ e IFSULDEMINAS $\left(S E_{I F}\right)$, respectivamente.

Por sua vez, a Figura 4, apresenta os resultados obtidos com as medições e simulações para as $24 \mathrm{~h}$ do dia 17 de julho e compara a resposta de ambos os modelos. O erro percentual dos valores simulados em relação aos valores medidos é apresentado na forma de barras.

Dessa maneira, tanto da Tabela III quanto da Figura 4, depreende-se que a simulação segue o comportamento esperado, apresentando resultados próximos aos dos dados medidos em campo para os casos estudados. Verifica-se ainda que os erros percentuais entre os valores simulados e medidos apresentados na Figura 4, estão, no geral, abaixo de $1,0 \%$ e, portanto, pode-se afirmar que a modelagem da rede é adequada para realização dos estudos almejados e reportados neste trabalho.

\section{METODOLOGIA DE CÁLCULO DE CH PROPOSTA}

Conforme definido em [11], a $\mathrm{CH}$ da rede para GD não é um cálculo fixo com um único resultado. Cada estudo pode adotar um parâmetro a ser analisado tais como, variações de tensão e/ou frequência [23], sobrecarga térmica [6], qualidade de energia e problemas de proteção [24]. De forma específica para este trabalho, utilizar-se-ão os parâmetros definidos pelo módulo 8 do PRODIST [25].

No entanto, segundo Karime et al. [5], o aumento de tensão é visto como o problema mais influente em um alimentador e, Kharrazi, Sreeram e Mishra [13] concluem que os problemas de regulação de tensão devido ao excesso de geração são mais prováveis de acontecer do que outros problemas de qualidade de energia e podem ocorrer mesmo em níveis de penetração mais baixos. Deste modo, neste trabalho, optou-se por calcular a $\mathrm{CH}$ da rede observando os limites de tensão em regime permanente. 
TABELA III

Validação de Dados do Modelo Computacional do Método Híbrido Proposto

\begin{tabular}{cccccccccccc}
\hline \multicolumn{3}{c|}{ Dados de Entrada } & \multicolumn{6}{c}{ Medido } & \multicolumn{4}{c}{ Simulado } \\
\hline Caso & dia & hora & $\begin{array}{c}P_{F V} \\
{[\mathrm{~kW}]}\end{array}$ & $\begin{array}{c}D_{I F} \\
{[\mathrm{~kW}]}\end{array}$ & $\begin{array}{c}D_{A L 19} \\
{[\mathrm{~kW}]}\end{array}$ & $\begin{array}{c}V_{S E_{P C 3}} \\
{[\mathrm{pu}]}\end{array}$ & $\begin{array}{c}V_{S E_{I F}} \\
{[\mathrm{pu}]}\end{array}$ & $\begin{array}{c}V_{S E_{P C 3}} \\
{[\mathrm{pu}]}\end{array}$ & $\begin{array}{c}\text { erro } \\
{[\%]}\end{array}$ & $\begin{array}{c}V_{S E_{I F}} \\
{[\mathrm{pu}]}\end{array}$ & $\begin{array}{c}\text { erro } \\
{[\%]}\end{array}$ \\
\hline 01 & 16.01 & $12 \mathrm{~h} 45$ & 54,43 & 0 & 2177,28 & 1,028 & 1,023 & 1,028 & 0,0000 & 1,02298 & 0,0020 \\
02 & 26.01 & $15 \mathrm{~h} 30$ & 52,56 & 0 & 2217,60 & 1,046 & 1,027 & 1,046 & 0,0000 & 1,02934 & 0,2240 \\
\hline 03 & 16.01 & $19 \mathrm{~h} 45$ & 0 & 9,74 & 3507,84 & 1,037 & 1,017 & 1,037 & 0,0000 & 1,0210 & 0,3933 \\
04 & 26.01 & $19 \mathrm{~h} 45$ & 0 & 9,41 & 3528,00 & 1,046 & 1,031 & 1,046 & 0,0000 & 1,0300 & 0,0970 \\
\hline
\end{tabular}

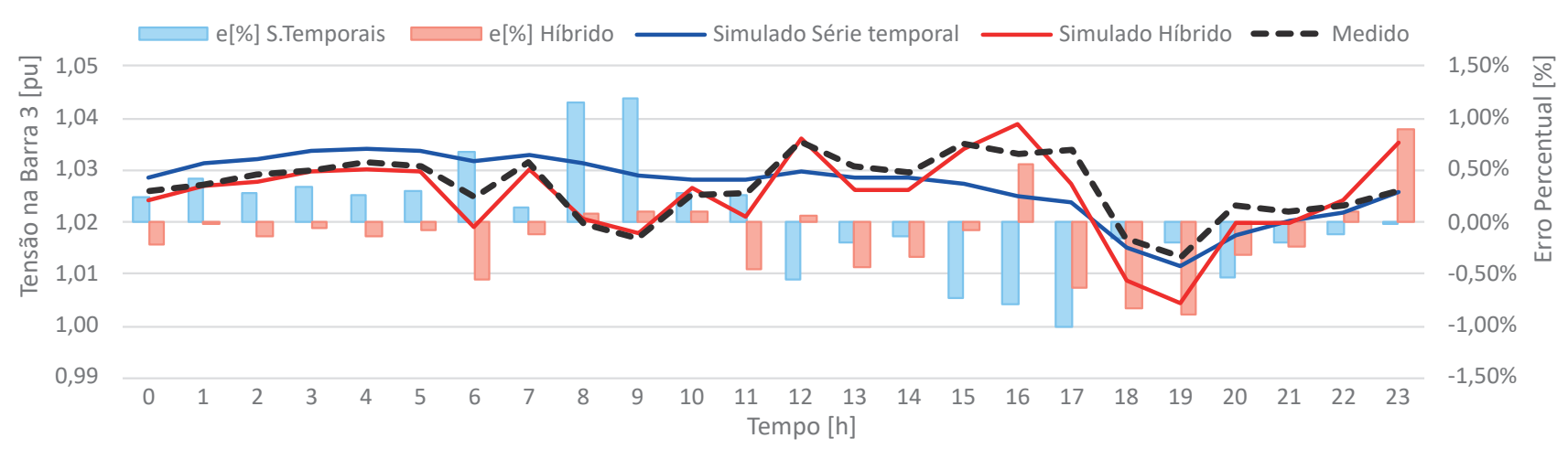

Fig. 4. Tensões na Barra 3 ao longo do dia 17.07.2019: Comparação entre valores medidos e simulados.

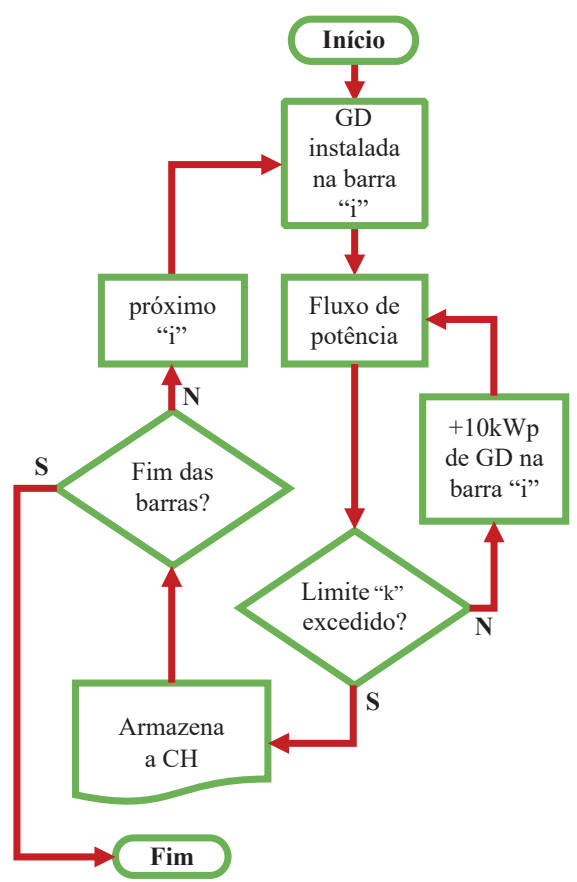

Fig. 5. Fluxograma simplificado de cálculo de $\mathrm{CH}$.

Assim sendo, para calcular a $\mathrm{CH}$ da rede baseado nos limites de tensão, o método híbrido se propõe a economizar tempo computacional ao simular o cenário mais crítico para a rede que ocorrerá sempre ao meio dia, com o pico de produção de energia fotovoltaica e baixo carregamento por parte das cargas. A identificação do cenário de interesse é possível ao se comparar as Figuras 2 e 3 e observar que o pico de produção de energia fotovoltaica (às $12 \mathrm{~h}$ ) não coincide com o pico de demanda do alimentador (19h).

Portanto, ao determinar a hora e o parâmetro do PRODIST para análise, o processo de cálculo pode ser escrito na forma do fluxograma da Figura 5 onde: $\boldsymbol{i}$ denota as barras que terão GD instaladas (Barras B1, B3, B5 e B6, ver Figura 1) e $\boldsymbol{k}$ indica o parâmetro analisado do PRODIST (no caso, variações de tensão de longa duração).

\section{RESULTADOS}

A análise computacional realizada primou pela implementação de cinco casos. O primeiro foi denominado de "caso base" onde o sistema não contará com nenhuma GD instalada. Nos casos seguintes, nas barras previamente selecionadas, foram adicionadas UFV's e o modelo que usa o método híbrido foi configurado para às $12 \mathrm{~h}$, isto é, pico de produção fotovoltaica e baixa demanda no alimentador 19 . Cada caso será confrontado com o modelo de séries temporais onde será simulado um período de $24 \mathrm{~h}$.

$\mathrm{Na}$ sequência são apresentados os principais resultados obtidos em cada caso analisado e por fim, determina-se a $\mathrm{CH}$ do alimentador.

\section{A. Caso Base}

Tem-se aqui o sistema operando sem nenhuma UFV instalada. Nessa configuração, as tensões das barras, ao meiodia, estão todas entre 1,016 e 1,031 pu como pode-se ver na Figura 6. Como era de se esperar, a tensão na barra $6\left(V_{B 6}\right)$ é a menor já que é a barra mais distante da subestação.

Ao observar o período de $24 \mathrm{~h}$, vê-se o comportamento típico de um alimentador radial predominantemente residencial, onde as tensões mais elevadas acontecem durante a madrugada e as mais baixas durante o pico de demanda que, neste caso, ocorre entre 18 e $19 \mathrm{~h}$.

\section{B. Caso 1}

Neste cenário a UFV será instalada na barra 6. A região onde essa barra se encontra é uma área de expansão do 


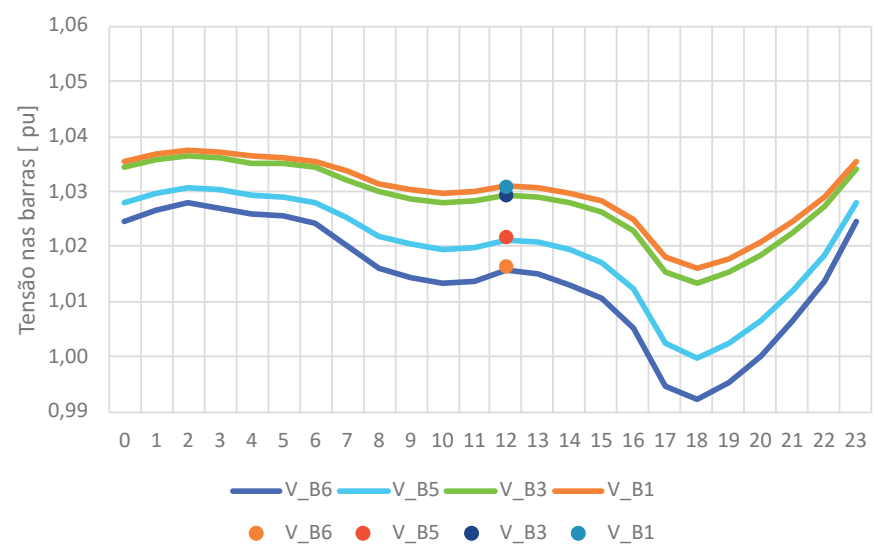

Fig. 6. Caso Base: Tensões nas Barras 6, 5, 3 e 1. As curvas são dadas pelo método de séries temporais e os Pontos, pelo método híbrido proposto.

município, possui grandes espaços para construções e pode servir a empreendimentos de mini usinas fotovoltaicas.

Seguindo os passos indicados na Figura 5, determinou-se que é possível instalar 2,3 MWp quando, então, extrapolou-se o limite de 1,05 pu estabelecido pelo PRODIST [25]. Observase na Figura 7 que a violação ocorre as $12 \mathrm{~h}$ no local da UFV, i.e., na barra 6 , conforme previsto, e que as tensões nos demais barramentos ficam entorno de 1,04 pu.

Pode-se ainda observar ao comparar as Figuras 6 e 7 que o comportamento do perfil de tensão é alterado com a inserção da UFV no final do alimentador.

\section{Caso 2}

A UFV, nesse caso, foi instalada na barra 5. A barra está situada em região de interesse da concessionária por se tratar de um grande adensamento populacional de classe média, predominantemente residencial, mas, que conta com escola, mercado e outros pequenos pontos comerciais.

De forma similar ao caso anterior, o limite superior de tensão foi rompido também pela barra na qual a UFV estava instalada comportando 3,5 MWp. Na Figura 8 é possível observar novamente que as demais barras também ficaram com o nível de tensão próximo de $1,05 \mathrm{pu}$. Isso pode ser explicado pelo fato da Barra 5 estar geograficamente próxima do centro do alimentador, impactando-o de forma mais uniforme.

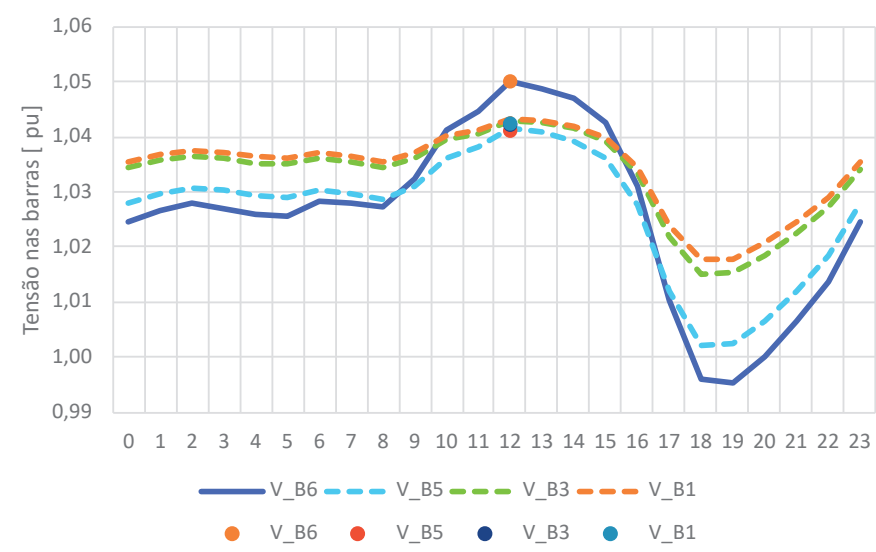

Fig. 7. Caso 01: UFV instalada na barra 6 (linha sólida do gráfico). As curvas são dadas pelo método de séries temporais e os Pontos, pelo método híbrido proposto.

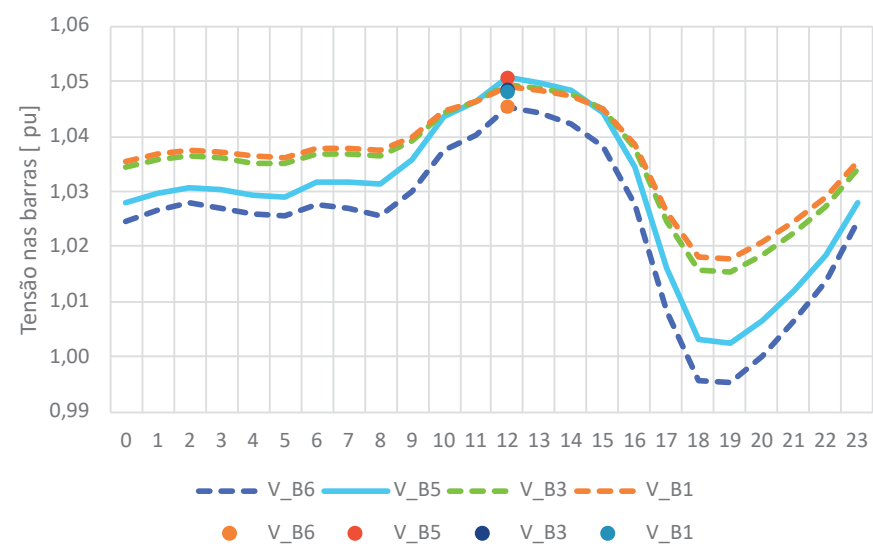

Fig. 8. Caso 02: UFV instalada na barra 5 (linha sólida do gráfico). As curvas são dadas pelo método de séries temporais e os Pontos, pelo método híbrido proposto.

\section{Caso 3}

O caso em questão, apresenta a barra 3 que atende um consumidor de média tensão (IFSULDEMINAS) que já possui GD instalada. Dentro da legislação atual (REN 482 [26]), esse único consumidor poderia expandir sua UFV até 5,0 MWp. Contudo, o limite superior de tensão já é excedido quando instalado um total de 3,8 MWp nesta barra, como pode-se observar na Figura 9. Ainda na Figura 9, vê-se que as barras 5 e 6 não foram tão afetadas quanto as barras 3 e 1, embora ainda apresentem elevação de tensão.

\section{E. Caso 4}

Nesse cenário, a UFV é instalada na barra 1. A barra está situada em uma região de grande adensamento populacional de classe média, também predominantemente residencial mas que conta com escola, mercado e outros pontos comerciais. Nesse aspecto, assemelha-se ao caso 2, no entanto, a diferença está no fato da barra 1 estar próxima da subestação.

Assim, o sistema comportou 3,9 MWp de GD instalada nesse barramento. Tal qual o caso anterior, vê-se que as barras 5 e 6 não foram tão afetadas quanto as barras 1 e 3, como pode-se observar na Figura 10.

A fim de melhor comparar os resultados entre a metodologia de série temporal e a metodologia híbrida proposta, a Tabela IV mostra os resultados obtidos e a variação

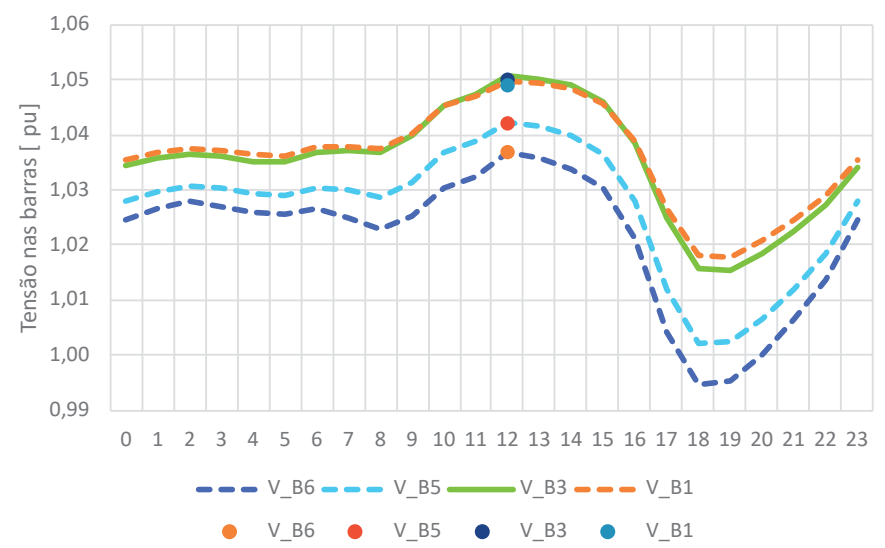

Fig. 9. Caso 03: UFV instalada na barra 3 (linha sólida do gráfico). As curvas são dadas pelo método de séries temporais e os Pontos, pelo método híbrido proposto. 


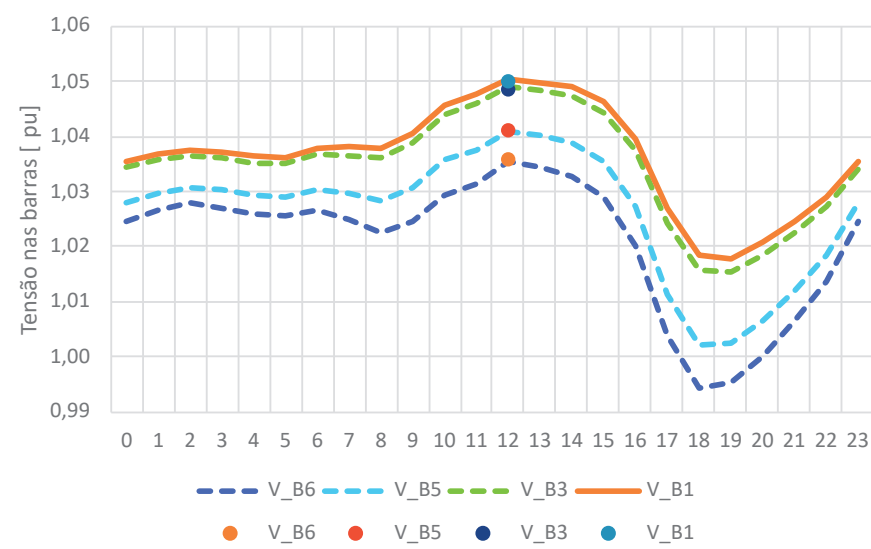

Fig. 10. Caso 04: UFV instalada na barra 1 (linha sólida do gráfico). As curvas são dadas pelo método de séries temporais e os Pontos, pelo método híbrido proposto.

percentual em relação ao método híbrido. Destaca-se que a variação entre elas ficaram abaixo de $0,1 \%$. Nos casos apresentados, percebe-se que a metodologia proposta chegou nos mesmos valores, com uma quantidade menor de casos simulados.

De posse dos resultados alcançados, pode-se ainda definir um Índice de Penetração Fotovoltaica $\left(\operatorname{Pen}_{F V}[\%]\right)$ como um percentual da demanda máxima do alimentador [27], conforme mostra a equação (3):

$$
\operatorname{Pen}_{F V}[\%]=\frac{P_{P_{U F V}}}{D_{\max _{A L}}} \cdot 100 \% .
$$

Onde:

$P_{P_{U F V}} \quad$ Potência pico da UFV;

$D_{\max } \quad$ Demanda máxima do alimentador.

Assim, adotando a $D_{\max _{A L}}$ igual a $4576,32 \mathrm{~kW}$ (conforme mostrado na Tabela III) e $P_{P_{U F V}}$ igual a $\mathrm{CH}$ obtida em cada caso simulado, obteve-se então índices de penetração de $51,4 \%, 76,5 \%, 81 \%$ e $85,7 \%$, respectivamente. O que vai ao encontro do postulado por Kharrazi, Sreeram e Mishra [13] mostrando que possíveis problemas de regulação de tensão devido ao excesso de geração podem ocorrer mesmo em níveis de penetração mais baixos.

Portanto, conclui-se que o estudo realizado revela que o alimentador em foco tem espaço para integração de geração distribuída, não obstante, deve-se atentar para a região de conexão pois, como observado, a distância em relação a subestação influenciará as demais barras. Dito de outra forma, é preferencial a conexão na barra 1, seguida pelas barras 3, 5 e por fim, a 6 . Como efeito colateral benéfico, desde que respeitados os limites, percebe-se a melhoria do nível de tensão no final do alimentador durante o período de funcionamento das UFV.

\section{CONCLUSÕES}

Em suma, o método híbrido apresentado utilizou uma série temporal com os dados de demanda do alimentador que é facilmente obtido em sua subestação de origem. As cargas de baixa tensão foram modeladas pela razão entre suas demandas máximas e a demanda máxima do alimentador, facilitando a implementação inicial do modelo.

\begin{tabular}{|c|c|c|c|c|}
\hline Com & ara & $\begin{array}{r}\text { TABEL } \\
\text { o entre Met } \\
\text { nsões nas Ba }\end{array}$ & $\begin{array}{l}\text { [V } \\
\text { ologias Aplica } \\
\text { as às 12h }\end{array}$ & las: \\
\hline & & $\begin{array}{c}\text { Mét. } \\
\text { Séries temporais } \\
{[\mathrm{pu}]}\end{array}$ & $\begin{array}{l}\text { Mét. } \\
\text { Híbrido proposto } \\
{[\mathrm{pu}]}\end{array}$ & $\Delta \%$ \\
\hline & $V_{B 6}$ & 1,0156 & 1,0164 & $-0,075 \%$ \\
\hline Caso Base & $V_{B 5}$ & 1,0212 & 1,0217 & $-0,050 \%$ \\
\hline & $V_{B 3}$ & 1,0293 & 1,0293 & $-0,004 \%$ \\
\hline & $V_{B 1}$ & 1,0309 & 1,0309 & $0,005 \%$ \\
\hline & $V_{B 6}$ & 1,0501 & 1,0501 & $0,000 \%$ \\
\hline Caso 01 & $V_{B 5}$ & 1,0414 & 1,0411 & $0,030 \%$ \\
\hline & $V_{B 3}$ & 1,0430 & 1,0422 & $0,077 \%$ \\
\hline & $\overline{V_{B 1}}$ & 1,0434 & 1,0424 & $0,087 \%$ \\
\hline & $V_{B 6}$ & 1,0453 & 1,0453 & $-0,018 \%$ \\
\hline Caso 02 & $V_{B 5}$ & 1,0507 & 1,0506 & $0,004 \%$ \\
\hline & $V_{B 3}$ & 1,0492 & 1,0485 & $0,070 \%$ \\
\hline & $V_{B 1}$ & 1,0490 & 1,0481 & $0,085 \%$ \\
\hline & $V_{B 6}$ & 1,0368 & 1,0369 & $-0,006 \%$ \\
\hline Caso 03 & $V_{B 5}$ & 1,0423 & 1,0421 & $0,017 \%$ \\
\hline & $V_{B 3}$ & 1,0506 & 1,0500 & $0,060 \%$ \\
\hline & $\overline{V_{B 1}}$ & 1,0498 & 1,0490 & $0,077 \%$ \\
\hline & $V_{B 6}$ & 1,0355 & 1,0359 & $-0,033 \%$ \\
\hline Caso 04 & $\overline{V_{B 5}}$ & 1,0410 & 1,0411 & $-0,010 \%$ \\
\hline & $\overline{V_{B 3}}$ & 1,0489 & 1,0485 & $0,035 \%$ \\
\hline & $V_{B 1}$ & 1,0505 & 1,0500 & $0,045 \%$ \\
\hline
\end{tabular}

Tendo em mente as dificuldades operacionais das concessionárias que veem o número de "pedidos de acesso" às suas redes crescerem exponencialmente ano após ano [3], faz com que a simulação apenas do ponto crítico do sistema agilize as análises e, consequentemente, a emissão dos pareceres de acesso para liberação de novas UFVs.

Assim, as vantagens da metodologia proposta podem ser resumidas em: (a) Obtenção dos parâmetros de entrada facilitada; (b) Agilidade na implementação do estudo; (c) Resultados coerentes com o método temporal.

Por fim, diante das vantagens supracitadas e da eficácia apresentada pelo método, a metodologia híbrida proposta se mostra como uma ferramenta promissora e atraente para os estudos de $\mathrm{CH}$ por parte das concessionárias.

\section{AGRADECIMENTOS}

Os autores agradecem a concessionária DMED pela disponibilidade e atenção e ao IFSULDEMINAS pelo suporte concedido à pesquisa. Este projeto foi financiado pelo projeto IFSolares (ANEEL PD-00051-0217/2017) parceria entre DMED e o IFSULDEMINAS Campus Poços de Caldas [4]. 


\section{REFERENCIAS}

[1] G. Masson, I. Kaizuka., Trends in photovoltaic applications 2019, 2019 ed., IEA, Paris, 2019, URL: www.iea-pvps.org.

[2] EPE, Balanço Energético Nacional 2019 - Relatório Síntese Ano Base 2018, EPE, 2019.

[3] ABSOLAR, Infográfico da Energia Solar Fotovoltaica no Brasil, ABSOLAR, ago 2020, URL: http://www. absolar.org.br/infografico-absolar.html.

[4] ANEEL/SPE, Chamada $N^{o}$. 001/2016 Projeto Prioritário de eficiência energética e estratégico de P\&D: "Eficiência Energética e Minigeração em Instituições Públicas de Educação Superior ”, ANEEL, Brasília, 2016, URL: http://bit.ly/38C2CPl.

[5] M. Karimi, H. Mokhlis, K. Naidu, S. Uddin, A. H. Bakar, "Photovoltaic penetration issues and impacts in distribution network - A review", Renewable and Sustainable Energy Reviews, vol. 53, pp. 594-605, 2016, doi:10.1016/j.rser.2015.08.042, URL: http://dx. doi.org/10.1016/j.rser.2015.08.042.

[6] P. M. Reinaldo, B. Scortegagna Dupczak, E. A. C. Aranha Neto, "Impact Assessment of Photovoltaic Distributed Generation on Celesc Low-Voltage Network", Eletrônica de Potência, vol. 25, no. 3, pp. 1-10, sep 2020, doi:10.18618/REP.2020.3.0013, URL: http://dx.doi.org/10.18618/REP.2020.3.0013.

[7] D. Chathurangi, U. Jayatunga, M. Rathnayake, A. Wickramasinghe, A. Agalgaonkar, S. Perera, "Potential power quality impacts on LV distribution networks with high penetration levels of solar PV", in 2018 18th International Conference on Harmonics and Quality of Power (ICHQP), vol. 2018-May, pp. 1-6, IEEE, may 2018, doi:10.1109/ICHQP.2018.8378890, URL: https://ieeexplore.ieee.org/document/8378890/.

[8] Joint Working Group C4.24/CIRED, Power Quality and EMC Issues with Future Electricity Networks, CIGRÉ, 2018.

[9] EPRI, Impact Factors, Methods, and Considerations for Calculating and Applying Hosting Capacity, EPRI, Palo Alto, 2018.

[10] A. Barin, L. Felipe Pozzatti, R. Quadros Machado, L. Neves Canha, F. Alberto Farret, A. da Rosa Abaide, "Multicriteria Analysis of Impacts of Distributed Generation Sources on Operational Network Characteristics for Distribution System Planning Concerning Steady-state and Transient Operations", Eletrônica de Potência, vol. 14, no. 2, pp. 75-83, may 2009, doi:10.18618/REP.2009.2.075083, URL: http://dx.doi.org/10.18618/REP.2009.2.075083.

[11] S. M. Ismael, S. H. Abdel Aleem, A. Y. Abdelaziz, A. F. Zobaa, "State-of-the-art of hosting capacity in modern power systems with distributed generation", Renewable Energy, vol. 130, pp. 1002-1020, jan 2019, doi:10.1016/j.renene.2018.07.008, URL: https: //doi.org/10.1016/j.renene.2018.07.008.

[12] E. Mulenga, M. H. Bollen, N. Etherden, "A review of hosting capacity quantification methods for photovoltaics in low-voltage distribution grids",
International Journal of Electrical Power \& Energy Systems, vol. 115, no. February 2019, p. 105445, feb 2020, doi:10.1016/j.ijepes.2019.105445, URL: https: //doi.org/10.1016/j.ijepes.2019.105445.

[13] A. Kharrazi, V. Sreeram, Y. Mishra, "Assessment techniques of the impact of grid-tied rooftop photovoltaic generation on the power quality of low voltage distribution network - A review", Renewable and Sustainable Energy Reviews, vol. 120, no. November 2019, p. 109643, mar 2020, doi:10.1016/j.rser.2019.109643, URL: https://doi.org/10.1016/j.rser.2019.109643.

[14] F. J. Von Zuben, Fundamentos para Processos Estocásticos, UNICAMP, Campinas, 2003.

[15] R. M. P. Gontijo, Alocação de Geradores Distribuídos Orientada por Fluxo de Potência Probabilístico, Monografia (graduação em engenharia elétrica), UFOP, João Monlevade, 2017, URL: http://www. monografias.ufop.br/handle/35400000/860.

[16] S. F. de Paula Silva, SSEP: Simulador de Sistemas Elétricos de Potência, 4.0.0.25 ed., Quality Engenharia \& Sistemas, Uberlândia, 2016.

[17] R. C. Dugan, D. Montenegro, A. Ballanti, Reference Guide: The Open Distribution System Simulator (OpenDSS), June, EPRI, Palo Alto, 2020.

[18] N. Kagan, C. C. B. de Oliveira, E. J. Robba, Introdução aos sistemas de distribuição de energia elétrica, 2 ed., Edgard Blücher, São Paulo, 2010.

[19] J. Jardini, C. Tahan, M. Gouvea, S. Ahn, F. Figueiredo, "Curvas de Carga Típicas e Agregação dos Consumidores Residenciais, Comerciais e Industriais de Baixa Tensão", in 4to Encontro Luso-AfroBrasileiro de Planjamento e Exploraçao de Redes de Energia - ELAB, pp. 391-397, ELAB, Rio de Janeiro, 1999.

[20] M. D. S. Marques, Metodologia para Modelagem de Curvas Típicas de Demanda Elétrica Utilizando Redes Neurais Artificiais Considerando Variáveis Climáticas, Dissertação, UNIVERSIDADE FEDERAL DO PAMPA, 2014.

[21] G. M. d. F. Paula, Curvas típicas de carga para o planejamento operacional do sistema de distribuição, Tese de Doutorado, USP, São Paulo, 2006.

[22] L. F. Sauthier, A. T. Diefenthäler, P. S. Sausen, A. T. Z. R. Sausen, "Modelagem Matemática de Módulos Fotovoltaicos Empregados em Ambientes de Simulação de Geração Distribuída", in 11th SEMINAR ON POWER ELECTRONICS AND CONTROL, p. 6, UFSM, Santa Maria, 2018, URL: https://www.ufsm. br/cursos/pos-graduacao/santa-maria/ppgee/papers/.

[23] M. Brito, M. Alves, L. Sampaio, C. Canesin, "Antiisland Strategies Applied At Distributed Generation Systems", Eletrônica de Potência, vol. 23, no. 2, pp. 226-234, apr 2018, doi:10.18618/REP.2018.2.2773, URL: http://dx.doi.org/10.18618/REP.2018.2.2773.

[24] D. Iglesias Brandão, F. Pinhabel Marafão, F. Alberto Farret, M. Godoy Simões, "Automatic Management Methodology For Photovoltaic Distributed Generation Systems", Eletrônica de Potência, vol. 18, no. 4, 
pp. 1257-1265, nov 2013, doi:10.18618/REP.2013. 4.12571265, URL: http://dx.doi.org/10.18618/REP. 2013.4.12571265.

[25] ANEEL, "Módulo 8 - Qualidade da Energia Elétrica", in Procedimentos de Distribuição de Energia Elétrica no Sistema Elétrico Nacional - PRODIST, chap. 8, p. 88, Agência Nacional de Energia Elétrica, Brasilia, 10 ed., 2018, URL: https://www.aneel.gov. br/documents/656827/14866914/M\{́́\}dulo\{_\}8Revis $\{\sim\{a\}\}$ o \{_\}10/2f7cb862-e9d7-3295-729ab619ac6baab9.

[26] ANEEL, Resolução Normativa $n^{\circ} 482$ de 17 de Abril de 2012, ANEEL, Brasilia, 2012, URL: http://www2. aneel.gov.br/cedoc/ren2012482.pdf.

[27] R. A. Kordkheili, B. Bak-Jensen, J. R-Pillai, P. Mahat, "Determining maximum photovoltaic penetration in a distribution grid considering grid operation limits", in 2014 IEEE PES General Meeting | Conference \& Exposition, vol. 2014-Octob, pp. 1-5, IEEE, jul 2014, doi:10.1109/PESGM.2014.6939292, URL: http: //ieeexplore.ieee.org/document/6939292/.

\section{DADOS BIOGRÁFICOS}

Ezequiel Junio de Lima, nasceu em 1985 em Belo Horizonte, é engenheiro eletricista (2010), mestre (2014) e doutorando em Engenharia Elétrica pela Universidade Federal Uberlândia. Atuou como engenheiro eletricista da Odebrecht Engenharia Industrial, foi sócio da empresa Central Construtora Ltda e hoje atua como professor do Instituto Federal do Sul de Minas Gerais (IFSULDEMINAS), campus Poços de Caldas. Suas temáticas de pesquisa são distorções harmônicas, estudos de potência elétrica em regime não senoidal, álgebra de Clifford, energia solar fotovoltaica e projetos de instalações elétricas.

Luiz Carlos Gomes de Freitas nasceu em Uberlândia-MG, em 1976. Possui graduação em Engenharia Elétrica com ênfase em Eletrotécnica (2001) pela Universidade Federal de Uberlândia, instituição onde também obteve os títulos de mestre e doutor (2003 e 2006, respectivamente) e da qual é professor efetivo atualmente. Atua na coordenação e orientação direta de trabalhos no Núcleo de Pesquisa em Eletrônica de Potência. Suas temáticas de pesquisas estão relacionadas às técnicas de correção ativa de fator de potência, retificadores híbridos, qualidade da energia, conversores estáticos e técnicas de controle em sistemas alimentados por fontes renováveis e microrredes, energia fotovoltaica. Em 2012 recebeu o Prize Paper Award da IEEE Industry Society Application Society - Industrial Automation and Control Committee pelas contribuições no campo de retificadores híbridos. 